\title{
Equality Sets for Recursively Enumerable Languages
}

\section{Vesa Halava}

\section{Tero Harju}

Department of Mathematics, University of Turku FIN-20014 Turku, Finland and TUCS - Turku Centre for Computer Science

E-mail: \{vehalava,harju\}@utu.fi

\section{Hendrik Jan Hoogeboom}

Department of Computer Science, Leiden University

P.O. Box 9512, 2300 RA Leiden, The Netherlands

E-mail: hoogeboom@liacs.nl

\section{Michel Latteux}

Université des Sciences et Technologies de Lille

Bâtiment M3, 59655 Villeneuve d'Ascq Cédex, FRANCE

E-mail:latteux@lifl.fr

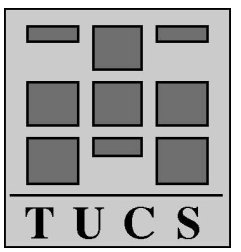

Turku Centre for Computer Science

TUCS Technical Report No 592

February 2004

ISBN 952-12-1309-4

ISSN 1239-1891 


\begin{abstract}
We consider shifted equality sets of the form $E_{G}\left(a, g_{1}, g_{2}\right)=\left\{w \mid g_{1}(w)=\right.$ $\left.a g_{2}(w)\right\}$, where $g_{1}$ and $g_{2}$ are nonerasing morphisms and $a$ is a letter. We are interested in the family consisting of the languages $h\left(E_{G}(J)\right)$, where $h$ is a coding and $E_{G}(J)$ is a shifted equality set. We prove several closure properties for this family. Moreover, we show that every every recursively enumerable language $L \subseteq A^{*}$ is a projection of a shifted equality set, that is, $L=\pi_{A}\left(E_{G}\left(a, g_{1}, g_{2}\right)\right)$ for some (nonerasing) morphisms $g_{1}$ and $g_{2}$ and a letter $a$, where $\pi_{A}$ deletes the letters not in $A$. Then we deduce that recursively enumerable star languages coincide with the projections of equality sets
\end{abstract}

Keywords: equality set, Post Correspondence Problem, coding, recursive enumerable set, projection

TUCS Laboratory

Discrete Mathematics for Information Technology 


\section{Introduction}

In formal language theory, languages are often determined by their generating grammars or accepting machines. It is also customary to say that languages generated by grammars of certain form or accepted by automata of specific type form a language family. Here we shall study a language family defined by simple generalized equality sets of the form $E_{G}(J)$, where $J=\left(a, g_{1}, g_{2}\right)$ is an instance of the shifted Post Correspondence Problem consisting of a letter $a$ and two morphisms $g_{1}$ and $g_{2}$. Then the set $E_{G}(J)$ consists of the words $w$ that satisfy $g_{1}(w)=a g_{2}(w)$.

Our motivation for these generalized equality sets comes partly from a result of [6], where it was proved that the family of regular valence languages is equal to the family of languages of the form $h\left(E_{G}(J)\right)$, where $h$ is a coding (i.e., a letter-to-letter morphism), and, moreover, in the instance $J=\left(a, g_{1}, g_{2}\right)$ the morphism $g_{2}$ is periodic. Here we shall consider general case where we do not assume $g_{2}$ to be periodic. However, we do assume that both morphisms to be nonerasing. We study the properties of this family $\mathcal{E} \mathcal{E}$ of languages by studying its closure properties. In particular, we show that $\mathcal{E} \mathcal{E}$ is closed under union, product, Kleene plus, intersection with regular sets. Also, more surprisingly, $\mathcal{E} \mathcal{E}$ is closed under nonerasing inverse morphisms.

In the last section, we consider the connection of the sifted equality sets to recursively enumerable languages. In particular, we show that every every recursively enumerable language $L \subseteq A^{*}$ is a projection of a shifted equality set, that is, $L=\pi_{A}\left(E_{G}\left(a, g_{1}, g_{2}\right)\right)$ for some (nonerasing) morphisms $g_{1}$ and $g_{2}$ and a letter $a$, where $\pi_{A}$ deletes the letters not in $A$.

The results of Sections 2 and 3 have been proved in the authors' conference paper [7]. The characterization results of Section 4 concerning presentation of recursively enumerable sets by shifted equality sets are new. The problem of presenting recursively enumerable sets using (general) equality sets was initiated by Salomaa [14], Culik [1], and Engelfriet and Rozenberg [3]; see also [4, 11, 15, 16].

\section{Preliminaries}

Let $A$ be an alphabet, and denote by $A^{*}$ the monoid of all finite words under the operation of concatenation. Note that the empty word, denoted by $\varepsilon$, is in the monoid $A^{*}$. The semigroup $A^{*} \backslash\{\varepsilon\}$ generated by $A$ is denoted by $A^{+}$. For a subset $L \subseteq A^{*}$, we denote by $L^{+}$the set of all words of the form $w_{1} w_{2} \ldots w_{n}$ for $w_{i} \in L$ with $n \geq 1$. Then $L^{*}=L^{+} \cup\{\varepsilon\}$.

For two words $u, v \in A^{*}, u$ is a prefix of $v$ if there exists a word $z \in A^{*}$ such that $v=u z$. If $v=u z$, then we also write $u=v z^{-1}$ and $z=u^{-1} v$.

In the following, let $A$ and $B$ be alphabets and $g: A^{*} \rightarrow B^{*}$ a mapping. For a word $x \in B^{*}$, we denote by $g^{-1}(x)=\left\{w \in A^{*} \mid g(w)=x\right\}$ the inverse 
image of $x$ under $g$. Then $g^{-1}(K)=\cup_{x \in K} g^{-1}(x)$ is the inverse image of $K \subseteq B^{*}$ under $g$, and $g(L)=\{g(w) \mid w \in L\}$ is the image of $L \subseteq A^{*}$ under $g$. Also, $g$ is a morphism if $g(u v)=g(u) g(v)$ for all $u, v \in A^{*}$. A morphism $g$ is a coding, if it maps letters to letters, that is, if $g(A) \subseteq B$. A morphism $g$ is said to be periodic, if there exists a word $w \in B^{*}$ such that $g\left(A^{*}\right) \subseteq\{w\}^{*}$.

If $A$ and $B$ are alphabets such that $A \subseteq B$, then the morphism $\pi_{A}: B^{*} \rightarrow$ $A^{*}$, which becomes defined by

$$
\pi_{A}(a)= \begin{cases}a & \text { if } a \in A, \\ \varepsilon & \text { if } a \in B \backslash A,\end{cases}
$$

is the projection of $B^{*}$ onto $A^{*}$.

In the following section, for a given alphabet $A$, the alphabet $\bar{A}=\{\bar{a} \mid$ $a \in A\}$ is a copy of $A$, if $A \cap \bar{A}=\emptyset$.

In the Post Correspondence Problem, PCP for short, we are given two morphisms $g_{1}, g_{2}: A^{*} \rightarrow B^{*}$ and it is asked whether or not there exists a nonempty word $w \in A^{+}$such that $g_{1}(w)=g_{2}(w)$. Here the pair $\left(g_{1}, g_{2}\right)$ is an instance of the PCP, and the word $w$ is called a solution. As a general reference to the problems and results concerning the Post Correspondence Problem, we give [8].

For an instance $I=\left(g_{1}, g_{2}\right)$ of the PCP, let

$$
E(I)=\left\{w \in A^{*} \mid g_{1}(w)=g_{2}(w)\right\}
$$

be its equality set. It is easy to show that an equality set $E=E\left(g_{1}, g_{2}\right)$ is always a monoid, that is, $E=E^{*}$. In fact, it is a free monoid, and thus the algebraic structure of $E$ is relatively simple, although the problem whether or not $E$ is trivial is undecidable.

We shall now consider special instances of the generalized Post Correspondence Problem in order to have slightly more structured equality sets. In the shifted Post Correspondence Problem, or shifted PCP for short, we are given two morphisms $g_{1}, g_{2}: A^{*} \rightarrow B^{*}$ and a letter $a \in B$, and it is asked whether there exists a word $w \in A^{*}$ such that

$$
g_{1}(w)=a g_{2}(w)
$$

The triple $J=\left(a, g_{1}, g_{2}\right)$ is called an instance of the shifted PCP and a word $w$ satisfying equation (2.1) is called a solution of $J$. It is clear that a solution $w$ is always nonempty. We let

$$
E_{G}(J)=\left\{w \in A^{+} \mid g_{1}(w)=a g_{2}(w)\right\}
$$

be the generalized equality set of $J$.

We shall denote by $\mathcal{C} \mathcal{E}$ the set of all languages $h\left(E_{G}(J)\right)$, where $h$ is a coding, and the morphisms in the instances $J$ of the shifted PCP are both nonerasing. 
In [6] $\mathcal{e} \mathcal{E}_{\text {per }}$ is defined as the family of languages $h\left(E_{G}(J)\right)$, where $h$ is a coding, and one of the morphisms in the instance $\mathrm{J}$ of the shifted PCP is assumed to be periodic. It was proved in [6] that $\mathcal{e} \mathcal{E}_{\text {per }}$ is equal to the family of languages defined by the regular valence grammars (see [12]). It is easy to see that the morphisms in the instances could have been assumed to be nonerasing in order to get the same result. Therefore, the family $\mathcal{E} \mathcal{E}$ studied in this paper is a generalization of $\mathcal{C} \mathcal{E}_{\text {per }}$ or, actually, $\mathcal{e} \mathcal{E}_{\text {per }}$ is a subfamily of $\mathcal{e} \mathcal{E}$.

\section{Closure properties of $\mathcal{E} \mathcal{E}$}

The closure properties of the family $\mathcal{e} \mathcal{E}_{\text {per }}$ follow from the known closure properties of regular valence languages. In this section, we study the closure properties of the more general family $\mathcal{C} \mathcal{E}$ under various operations.

Before we start our journey through the closure results, we make first some assumptions of the instances of the shifted PCP defining the languages at hand.

An instance $J=\left(a, g_{1}, g_{2}\right)$ of the shifted PCP is said to be frontal, if the shift letter $a$ appears only as the first letter in the images of $g_{1}$ and $a$ does not occur at all in the images of $g_{2}$.

Lemma 1. Let $L=h\left(E_{G}(J)\right)$ for a instance $J=\left(a, g_{1}, g_{2}\right)$ of the shifted $P C P$ and a coding $h$. There exists a frontal instance $J^{\prime}=\left(\#, g_{1}^{\prime}, g_{2}^{\prime}\right)$ and a coding $h^{\prime}$ such that $L=h^{\prime}\left(E_{G}\left(J^{\prime}\right)\right)$.

Proof. Assume $g_{1}, g_{2}: A^{*} \rightarrow B^{*}$ and $h: A^{*} \rightarrow C^{*}$. Let \# be a letter not in $B$. We shall construct a new instance $J^{\prime}=\left(\#, g_{1}^{\prime}, g_{2}^{\prime}\right)$, where $g_{1}^{\prime}, g_{2}^{\prime}:(A \cup \bar{A})^{*} \rightarrow$ $(B \cup\{\#\})^{*}$ and $\bar{A}$ is a copy of $A$, by setting for all $x \in A g_{2}^{\prime}(x)=g_{2}^{\prime}(\bar{x})=$ $g_{2}(x)$, and $g_{1}^{\prime}(x)=g_{1}(x)$ and

$$
g_{1}^{\prime}(\bar{x})= \begin{cases}g_{1}(x), & \text { if } a \text { is not a prefix of } g_{1}(x), \\ \# w, & \text { if } g_{1}(x)=a w .\end{cases}
$$

Define a new coding $h^{\prime}:(A \cup \bar{A})^{*} \rightarrow C^{*}$ by $h^{\prime}(x)=h^{\prime}(\bar{x})=h(x)$ for all $x \in A$. It is now obvious that $L=h^{\prime}\left(E_{G}\left(J^{\prime}\right)\right)$.

The next lemma shows that we may also assume that the instance $\left(g_{1}, g_{2}\right)$ does not have any nontrivial solutions, that is, $E\left(g_{1}, g_{2}\right)=\{\varepsilon\}$ for all instances $J=\left(a, g_{1}, g_{2}\right)$ defining the language $h\left(E_{G}(J)\right)$. For this result we introduce two mappings which are used for desynchronizing a pair of morphisms. Let $d$ be a new letter. For a word $u=a_{1} a_{2} \cdots a_{n}$, where each $a_{i}$ is a letter, define

$$
\ell_{d}(u)=d a_{1} d a_{2} d \cdots d a_{n} \quad \text { and } \quad r_{d}(u)=a_{1} d a_{2} d \cdots d a_{n} d .
$$

In other words, $\ell_{d}$ is a morphism that adds $d$ in front of every letter and $r_{d}$ is a morphism that adds $d$ after every letter of a word. 
Lemma 2. For every instance $J$ of the shifted PCP and coding $h$, there exists a frontal instance $J^{\prime}=\left(a, g_{1}^{\prime}, g_{2}^{\prime}\right)$ and a coding $h^{\prime}$ such that $h\left(E_{G}(J)\right)=$ $h^{\prime}\left(E_{G}\left(J^{\prime}\right)\right)$ and $E\left(g_{1}^{\prime}, g_{2}^{\prime}\right)=\{\varepsilon\}$.

Proof. By Lemma 1, we can assume that $J=\left(a, g_{1}, g_{2}\right)$ is a frontal instance of the shifted PCP. Let $g_{1}, g_{2}: A^{*} \rightarrow B^{*}$, and let $h: A^{*} \rightarrow C^{*}$. We define new morphisms $g_{1}^{\prime}, g_{2}^{\prime}:(A \cup \bar{A})^{*} \rightarrow(B \cup\{d\})^{*}$, where $d \notin B$ is a new letter and $\bar{A}$ is a copy of $A$, as follows. For all $x \in A$,

$$
\begin{aligned}
& g_{2}^{\prime}(x)=\ell_{d}\left(g_{2}(x)\right) \quad \text { and } \quad g_{2}^{\prime}(\bar{x})=\ell_{d}\left(g_{2}(x)\right) d, \\
& g_{1}^{\prime}(x)=g_{1}^{\prime}(\bar{x})= \begin{cases}a d \cdot r_{d}(w), & \text { if } g_{1}(x)=a w, \\
r_{d}\left(g_{1}(x)\right), & \text { if } a \text { is not a prefix of } g_{1}(x) .\end{cases}
\end{aligned}
$$

It is clear that $J^{\prime}$ is a frontal instance. Note also that, since the images $g_{2}^{\prime}(\bar{x})$ start and end in $d$, the letters in $\bar{A}$ can be used only as the last letter of a solution of $J^{\prime}=\left(a, g_{1}^{\prime}, g_{2}^{\prime}\right)$. Since every image by $g_{2}^{\prime}$ begins with letter $d$ and it is not a prefix of any image of $g_{1}^{\prime}$, we obtain that $E\left(g_{1}^{\prime}, g_{2}^{\prime}\right)=$ $\{\varepsilon\}$. On the other hand, $\left(a, g_{1}^{\prime}, g_{2}^{\prime}\right)$ has a solution $w \bar{x}$ if and only if $w x$ is a solution of $\left(a, g_{1}, g_{2}\right)$. Therefore, we can define $h^{\prime}:(A \cup \bar{A})^{*} \rightarrow C^{*}$ by $h^{\prime}(x)=h^{\prime}(\bar{x})=h(x)$ for all $x \in A$. The claim of the lemma follows, since obviously $h\left(E_{G}(J)\right)=h^{\prime}\left(E_{G}\left(J^{\prime}\right)\right)$.

We call an instance $\left(a, g_{1}, g_{2}\right)$ reduced, if it is frontal and $E\left(g_{1}, g_{2}\right)=\{\varepsilon\}$.

\subsection{Union and product}

Theorem 3. The family $\mathcal{E} \mathcal{E}$ is closed under union and product of languages.

Proof. Let $K, L \in \mathcal{C} \mathcal{E}$ with $K=h_{1}\left(E_{G}\left(J_{1}\right)\right)$ and $L=h_{2}\left(E_{G}\left(J_{2}\right)\right)$, where $J_{1}=\left(a_{1}, g_{11}, g_{12}\right)$ and $J_{2}=\left(a_{2}, g_{21}, g_{22}\right)$ are reduced, and $g_{11}, g_{12}: \Sigma^{*} \rightarrow B_{1}^{*}$ and $g_{21}, g_{22}: \Omega^{*} \rightarrow B_{2}^{*}$. Without restriction we can suppose that $\Omega \cap \Sigma=\emptyset$. (Otherwise we take a copy of the alphabet $\Omega$ that is disjoint from $\Sigma$.) We can also assume that $B_{1} \cap B_{2}=\emptyset$. Let $B=B_{1} \cup B_{2}$.

(1) For the closure under union, let \# be a new letter. First replace every appearance of the shift letters $a_{1}$ and $a_{2}$ in $J_{1}$ and $J_{2}$ by \#. Define $g_{1}, g_{2}:(\Sigma \cup \Omega)^{*} \rightarrow B^{*}$ as follows: for all $x \in \Sigma \cup \Omega$,

$$
g_{1}(x)=\left\{\begin{array}{ll}
g_{11}(x), & \text { if } x \in \Sigma \\
g_{21}(x), & \text { if } x \in \Omega
\end{array} \quad \text { and } \quad g_{2}(x)= \begin{cases}g_{12}(x), & \text { if } x \in \Sigma \\
g_{22}(x), & \text { if } x \in \Omega .\end{cases}\right.
$$

Define a coding $h:(\Sigma \cup \Omega)^{*} \rightarrow C^{*}$ similarly:

$$
h(x)= \begin{cases}h_{1}(x), & \text { if } x \in \Sigma \\ h_{2}(x), & \text { if } x \in \Omega\end{cases}
$$


Since $\Sigma \cap \Omega=\emptyset$, and the instances $J_{1}$ and $J_{2}$ are reduced (i.e., $E\left(g_{11}, g_{12}\right)=$ $\left.\{\varepsilon\}=E\left(g_{21}, g_{22}\right)\right)$, it follows that the solutions in $E_{G}\left(J_{1}\right)$ and $E_{G}\left(J_{2}\right)$ cannot be combined or mixed. Thus, it is easy to see that $h\left(E_{G}\left(\#, g_{1}, g_{2}\right)\right)=K \cup L$.

(2) For the closure under product, we assume that the length of the images of the morphisms are at least 2. (Actually, this is needed only for $\left.g_{11}\right)$. This can be assumed, for example, by the construction in the proof of Lemma 2.

We shall prove that $K L=\{u v \mid u \in K, v \in L\}$ is in $\mathcal{C} \mathcal{E}$. For this, we define $g_{1}, g_{2}:(\Sigma \cup \Omega)^{*} \rightarrow B^{*}$ in the following way: for each $x \in \Sigma$,

$$
g_{1}(x)= \begin{cases}\ell_{a_{2}}\left(g_{11}(x)\right), & \text { if } a_{1} \text { is not a prefix of } g_{11}(x), \\ a_{1} y \ell_{a_{2}}(w), & \text { if } g_{11}(x)=a_{1} y w \quad\left(y \in B_{1}\right)\end{cases}
$$

and

$$
g_{2}(x)=r_{a_{2}}\left(g_{12}(x)\right)
$$

and for each $x \in \Omega, g_{1}(x)=g_{21}(x)$ and $g_{2}(x)=g_{22}(x)$. If we now define $h$ by combining $h_{1}$ and $h_{2}$ as in (3.3), we obtain that $h\left(E_{G}\left(a_{1}, g_{1}, g_{2}\right)\right)=K L$.

We shall now extend the above result by proving that $\mathcal{E} \mathcal{E}$ is closed under Kleene plus, i.e., if $K \in \mathcal{C} \mathcal{E}$, then also $K^{+} \in \mathcal{C} \mathcal{E}$. Clearly $\mathcal{C} \mathcal{E}$ is not closed under Kleene star, since the empty word does not belong to any language in eE.

Theorem 4. The family $\mathcal{E} \mathcal{E}$ is closed under Kleene plus.

Proof. Let $K=h\left(E_{G}\left(a, g_{1}, g_{2}\right)\right)$, where $g_{1}, g_{2}: A^{*} \rightarrow B^{*}$ are nonerasing morphisms, $h: A^{*} \rightarrow C^{*}$ is a coding and the instance $\left(a, g_{1}, g_{2}\right)$ is frontal. Also, let $\bar{A}$ be a copy of $A$, and define $\bar{g}_{1}, \bar{g}_{2}:(A \cup \bar{A})^{*} \rightarrow B^{*}$ in the following way: for each $x \in A$,

$$
\begin{aligned}
& \bar{g}_{1}(x)=g_{1}(x) \text { and } \quad \bar{g}_{2}(x)=g_{2}(x), \\
& \bar{g}_{1}(\bar{x})=\left\{\begin{array}{l}
\ell_{a}\left(g_{1}(x)\right), \text { if } a \text { is not a prefix of } g_{1}(x), \\
\ell_{a}(w), \text { if } g_{1}(x)=a w,
\end{array}\right. \\
& \bar{g}_{2}(\bar{x})=r_{a}\left(g_{2}(x)\right) .
\end{aligned}
$$

Extend $h$ also to $\bar{A}$ by setting $h(\bar{x})=h(x)$ for all $x \in A$.

Now $h\left(E_{G}\left(a, \bar{g}_{1}, \bar{g}_{2}\right)\right)=K^{+}$, since $\bar{g}_{1}(w)=a \bar{g}_{2}(w)$ if and only if, $w=$ $x_{1} \cdots x_{n} x_{n+1}$, where $x_{i} \in \bar{A}^{+}$for $1 \leq i \leq n, x_{n+1} \in A^{+}, \bar{g}_{1}\left(x_{i}\right) a=a \bar{g}_{2}\left(x_{i}\right)$ for $1 \leq i \leq n$ and $\bar{g}_{1}\left(x_{n+1}\right)=a \bar{g}_{2}\left(x_{n+1}\right)$. After removing the bars form the letters $x_{i}$ (by $h$ ), we obtain words in $E_{G}\left(a, g_{1}, g_{2}\right)$. 


\subsection{Intersection with regular languages}

We show now that $\mathcal{E} \mathcal{E}$ is closed under intersections with regular languages.

Theorem 5. The family $\mathcal{E} \mathcal{E}$ is closed under intersections with regular languages.

Proof. Let $J=\left(a, g_{1}, g_{2}\right)$ be an instance of the shifted PCP, $g_{1}, g_{2}: \Sigma^{*} \rightarrow B^{*}$. Let $L=h\left(E_{G}(J)\right)$, where $h: \Sigma^{*} \rightarrow C^{*}$ is a coding.

We shall prove that $h\left(E_{G}(J)\right) \cap R$ is in $\mathcal{C} \mathcal{E}$ for all regular $R \subseteq B^{*}$. We note first that $h\left(E_{G}(J)\right) \cap R=h\left(E_{G}(J) \cap h^{-1}(R)\right)$, and therefore it is sufficient to show that, for all regular languages $R \subseteq \Sigma^{*}, h\left(E_{G}(J) \cap R\right)$ is in $\mathcal{E} \mathcal{E}$. Therefore, we shall give a construction for instances $J^{\prime}$ of the shifted PCP such that $E_{G}\left(J^{\prime}\right)=E_{G}(J) \cap R$.

Assume $R \subseteq \Sigma^{*}$ is regular, and let $G=(N, \Sigma, P, S)$ be a right linear grammar generating $R$ (see [13]). Let $N=\left\{A_{0}, \ldots, A_{n-1}\right\}$, where $S=A_{0}$, and assume without restriction, that $S$ does not appear on the right hand side of any production. We consider the set $P$ of the productions as an alphabet.

Let $\#$ and $d$ be new letters. We define $g_{1}^{\prime}, g_{2}^{\prime}: P^{*} \rightarrow(B \cup\{d, \#\})^{*}$ as follows. First assume that

$$
g_{1}(a)=a_{1} a_{2} \ldots a_{k} \quad \text { and } \quad g_{2}(a)=b_{1} b_{2} \ldots b_{m}
$$

for the (generic) letter $a$. We define

$$
g_{1}^{\prime}(p)= \begin{cases}\# d^{n} a_{1} d^{n} a_{2} d^{n} \ldots a_{k} d^{j}, & \text { if } p=\left(A_{0} \rightarrow a A_{j}\right) \\ d^{n-i} a_{1} d^{n} a_{2} d^{n} \ldots a_{k} d^{j}, & \text { if } p=\left(A_{i} \rightarrow a A_{j}\right), \\ \# d^{n} a_{1} d^{n} a_{2} d^{n} \ldots a_{k}, & \text { if } p=\left(A_{0} \rightarrow a\right), \\ d^{n-i} a_{1} d^{n} a_{2} d^{n} \ldots a_{k}, & \text { if } p=\left(A_{i} \rightarrow a\right) .\end{cases}
$$

and

$$
g_{2}^{\prime}(p)=d^{n} b_{1} d^{n} b_{2} \ldots d^{n} b_{m}, \quad \text { if } p=(A \rightarrow a X),
$$

where $X \in N \cup\{\varepsilon\}$.

As in [9], $E_{G}\left(J^{\prime}\right)=E_{G}(J) \cap R$ for the new instance $J^{\prime}=\left(\#, g_{1}^{\prime}, g_{2}^{\prime}\right)$. The claim follows from this.

\subsection{Morphisms}

Next we shall present a construction for the closure under nonerasing morphisms. This construction is a bit more complicated than the previous ones.

Theorem 6. The family $\mathcal{E} \mathcal{E}$ is closed under taking images of nonerasing morphisms. 
Proof. Let $J=\left(a, g_{1}, g_{2}\right)$ be an instance of the shifted PCP, where $g_{1}, g_{2}: A^{*} \rightarrow$ $B^{*}$. Let $L=h\left(E_{G}(J)\right)$, where $h: A^{*} \rightarrow C^{*}$ is a coding. Assume that $f: C^{*} \rightarrow \Sigma^{*}$ is a nonerasing morphism. We shall construct $h^{\prime}, g_{1}^{\prime}$ and $g_{2}^{\prime}$ such that $f(L)=h^{\prime}\left(E_{G}\left(J^{\prime}\right)\right)$ for the new instance $J^{\prime}=\left(a, g_{1}^{\prime}, g_{2}^{\prime}\right)$.

First we show that we can restrict ourselves to cases where

$$
\min \left\{\left|g_{1}(x)\right|,\left|g_{2}(x)\right|\right\} \geq|f(x)| \text { for all } x \in A .
$$

Indeed, suppose the instance $J$ does not satisfy (3.4). We construct a new instance $\bar{J}=\left(\#, \bar{g}_{1}, \bar{g}_{2}\right)$ and a coding $\bar{h}$ such that $\bar{h}\left(E_{G}(\bar{J})=h\left(E_{G}(J)\right)\right.$ and $\bar{g}_{1}$ and $\bar{g}_{2}$ do fulfill (3.4). Let $c \notin B$ be a new letter. Let $k=\max _{x \in A}\{|f(x)|\}$. We define $\bar{g}_{1}(x)=\ell_{c}^{k}\left(g_{1}(x)\right)$ and $\bar{g}_{2}(x)=\ell_{c}^{k}\left(g_{2}(x)\right)$ for all $x \in A$. We also need a new copy $x^{\prime}$ of each letter $x$ for which $a$ is a prefix of $g_{1}(x)$. If $g_{1}(x)=a w$, where $w \in B^{*}$, then define $\bar{g}_{1}\left(x^{\prime}\right)=\# \ell_{c}^{k}(w)$. It now follows that if $u \in E_{G}(\bar{J})$, then $u=x^{\prime} v$ for some word $v \in A^{*}$ and $x v \in E_{G}(J)$. Therefore, by defining $\bar{h}$ as follows

$$
\bar{h}(y)= \begin{cases}h(y), & \text { if } y \in A, \\ h(x), & \text { if } y=x^{\prime}\end{cases}
$$

we have $\bar{h}\left(E_{G}(\bar{J})=h\left(E_{G}(J)\right)\right.$ as required.

Now assume that (3.4) holds in $J=\left(a, g_{1}, g_{2}\right)$ and for $f$. Let us consider the nonerasing morphism $f h: A^{*} \rightarrow \Sigma^{*}$. Note that also the composition $f h$ satisfies (3.4). In order to prove the claim, it is clearly sufficient to consider the case, where $h$ is the identity mapping, that is, $f=f h$.

First we define for every image $f(x)$, where $x \in A$, a new alphabet $A_{x}=\left\{b_{x} \mid b \in \Sigma\right\}$. We consider the words

$$
\left(b_{1} b_{2} \ldots b_{m}\right)_{x}=\left(b_{1}\right)_{x}\left(b_{2}\right)_{x} \ldots\left(b_{m}\right)_{x}
$$

for $f(x)=b_{1} \ldots b_{m}$.

Let $c$ and $d$ be new letters and let $n=\sum_{x \in A}|f(x)|$. Assume that $A=\left\{x_{1}, x_{2}, \ldots, x_{q}\right\}$.

Partition the integers $1,2, \ldots, n$ into $q$ sets such that for the letter $x_{i}$ there corresponds a set, say $S_{i}=\left\{i_{1}, i_{2}, \ldots, i_{\mid f\left(x_{i} \mid\right.}\right\}$, of $\left|f\left(x_{i}\right)\right|$ integers.

Assume that $f\left(x_{i}\right)=b_{1} \ldots b_{m}, g_{1}\left(x_{i}\right)=a_{1} a_{2} \ldots a_{\ell}$, and $g_{2}\left(x_{i}\right)=a_{1}^{\prime} a_{2}^{\prime} \ldots a_{k}^{\prime}$. We define new morphisms $g_{1}^{\prime}$ and $g_{2}^{\prime}$ as follows:

$$
\begin{aligned}
g_{1}^{\prime}\left(\left(b_{1}\right)_{x_{i}}\right) & =c^{n} d^{n} a_{1} c^{i_{1}}, \\
g_{1}^{\prime}\left(\left(b_{j}\right)_{x_{i}}\right) & =c^{n-i_{j-1}} d^{n} a_{j} c^{i_{j}} \quad \text { for } j=2, \ldots, m-1, \\
g_{1}^{\prime}\left(\left(b_{m}\right)_{x_{i}}\right) & =c^{n-i_{m-1}} d^{n} a_{m} c^{n} d^{n} \ldots c^{n} d^{n} a_{\ell},
\end{aligned}
$$

and

$$
\begin{aligned}
g_{2}^{\prime}\left(\left(b_{1}\right)_{x_{i}}\right) & =c^{n} d^{n} a_{1} c^{n} d^{i_{1}}, \\
g_{2}^{\prime}\left(\left(b_{j}\right)_{x_{i}}\right) & =d^{n-i_{j-1}} a_{j}^{\prime} c^{n} d^{i_{j}} \quad \text { for } j=2, \ldots, m-1, \\
g_{2}^{\prime}\left(\left(b_{m}\right)_{x_{i}}\right) & =c^{n} d^{n-i_{m-1}} a_{m}^{\prime} c^{n} d^{n} \ldots c^{n} d^{n} a_{k}^{\prime} .
\end{aligned}
$$


Then

$$
\begin{aligned}
& g_{1}^{\prime}\left(\left(b_{1} \ldots b_{m}\right)_{x_{i}}\right)=c^{n} d^{n} a_{1} c^{n} d^{n} a_{2} \ldots c^{n} d^{n} a_{\ell}, \\
& g_{2}^{\prime}\left(\left(b_{1} \ldots b_{m}\right)_{x_{i}}\right)=c^{n} d^{n} a_{1}^{\prime} c^{n} d^{n} a_{2}^{\prime} \ldots c^{n} d^{n} a_{k}^{\prime} .
\end{aligned}
$$

The beginning has to be still fixed. For the cases, where $a_{1}=a$, we need new letters $\left(b_{1}\right)_{x_{i}}^{\prime}$, for which we define

$$
g_{1}^{\prime}\left(\left(b_{1}\right)_{x_{i}}^{\prime}\right)=a c^{i_{1}} \text { and } g_{2}^{\prime}\left(\left(b_{1}\right)_{x_{i}}^{\prime}\right)=c^{n} d^{n} a_{j} c^{n} d^{i_{1}} .
$$

Now our constructions for the morphisms $g_{1}^{\prime}$ and $g_{2}^{\prime}$ are completed.

Next we define $h^{\prime}$, by setting $h^{\prime}\left(\left(b_{i}\right)_{x}\right)=b_{i}$ and $h^{\prime}\left(\left(b_{1}\right)_{x}^{\prime}\right)=b_{1}$ for all $i$ and $x$. We obtain that $h^{\prime}\left(E_{G}\left(J^{\prime}\right)\right)=f\left(h\left(E_{G}(J)\right)\right.$, which proves the claim.

Next we shall prove that the family $\mathcal{C} \mathcal{E}$ is closed under inverse of nonerasing morphisms.

Theorem 7. The family $\mathcal{E} \mathcal{E}$ is closed under nonerasing inverse morphisms.

Proof. Consider an instance $h\left(E_{G}(J)\right)$, where $J=\left(a, g_{1}, g_{2}\right)$ with $g_{i}: A^{*} \rightarrow$ $B^{*}$ and $h: A^{*} \rightarrow C^{*}$ is a coding. We may assume that $h(A)=C$.

Moreover, let $g: \Sigma^{*} \rightarrow C^{*}$ be a nonerasing morphism.

For each $x \in \Sigma$, let $h^{-1} g(x)=\left\{v_{x, 1}, v_{x, 2}, \ldots, v_{x, k_{x}}\right\}$ and let

$$
\Sigma_{x}=\left\{x^{(1)}, \ldots, x^{\left(k_{x}\right)}\right\}
$$

be a set of new letters for $x$. Denote $\Theta=\cup_{x \in \Sigma} \Sigma_{x}$, and define the morphisms $g_{1}^{\prime}, g_{2}^{\prime}: \Theta^{*} \rightarrow B^{*}$ and the coding $t: \Theta^{*} \rightarrow \Sigma^{*}$ by

$$
g_{j}^{\prime}\left(x^{(i)}\right)=g_{j}\left(v_{x, i}\right) \text { for } j=1,2, \text { and } t\left(x^{(i)}\right)=x
$$

for each $x^{(i)} \in \Theta$.

Consider the instance $J^{\prime}=\left(a, g_{1}^{\prime}, g_{2}^{\prime}\right)$.

Now, assume that $u=a_{1} a_{2} \ldots a_{n} \in g^{-1} h\left(E_{G}(J)\right.$ ) (with $a_{i} \in \Sigma$ ). Then there exists a word $w=w_{1} w_{2} \ldots w_{n}$ such that $g_{1}(w)=a g_{2}(w)$ and $a_{i} \in$ $g^{-1} h\left(w_{i}\right)$, that is, $w_{i}=v_{a_{i}, r_{i}} \in h^{-1} g\left(a_{i}\right)$ for some $r_{i}$, and so $g_{1}^{\prime}\left(w^{\prime}\right)=$ $a g_{2}^{\prime}\left(w^{\prime}\right)$ for the word $w^{\prime}=a_{1}^{\left(r_{1}\right)} a_{2}^{\left(r_{2}\right)} \ldots a_{n}^{\left(r_{n}\right)}$, for which $t\left(w^{\prime}\right)=u$. Therefore $u \in t\left(E_{G}\left(J^{\prime}\right)\right)$.

The converse inclusion $t\left(E_{G}\left(J^{\prime}\right)\right) \subseteq g^{-1} h\left(E_{G}(J)\right)$ is clear by the above constructions.

Let $A$ and $B$ be two alphabets. A mapping $\tau: A^{*} \rightarrow 2^{B^{*}}$, where $2^{B^{*}}$ denotes the set of all subsets of $B^{*}$, is a substitution if for all $u, v \in A^{*}$

$$
\tau(u v)=\tau(u) \tau(v) .
$$

Note that $\tau$ is actually a morphism from $A^{*}$ to $2^{B^{*}}$.

A substitution $\tau$ is called finite if $\tau(a)$ is a finite set for all $a \in A$, and nonerasing if $\varepsilon \notin \tau(a)$ for all $a \in A$. 
Corollary 8. The family $\mathrm{C} \mathcal{E}$ is closed under nonerasing finite substitutions.

Proof. Since $\mathcal{E} \mathcal{E}$ is closed under nonerasing morphisms, inverses of nonerasing morphisms, it is closed under nonerasing finite substitutions. Indeed, as is immediate, every finite substitution is a composition of an inverse of a coding and a nonerasing morphism.

Note that $\mathcal{C} \mathcal{E}$ is almost a trio, see [5], but it seems that it is not closed under all inverse morphisms. It is also almost a bifaithful rational cone, see [10], but since the languages do not contain the empty word, $\mathcal{E} \mathcal{E}$ is not closed under the bifaithful finite transducers.

\section{Equality sets and recursively enumerable languages}

The following result of Engelfriet and Rozenberg [3] gives a classical morphic representation of recursively enumerable languages; see Salomaa [15] (see Theorem 6.9 , page 111 ). Recall that $\pi_{A}$ denotes the projection onto $A^{*}$.

Theorem 9. For every recursively enumerable language $L \subseteq A^{*}$, there are two morphisms $h_{1}, h_{2}$ and a regular language $R$ such that $L=\pi_{A}\left(E\left(h_{1}, h_{2}\right) \cap\right.$ $R)$.

A slight modification of its proof permits to strengthen this theorem:

Lemma 10. For every recursively enumerable language $L \subseteq A^{*}$, there are two nonerasing morphisms $h_{1}, h_{2}$ and a regular language $R$ such that $L=$ $\pi_{A}\left(E\left(h_{1}, h_{2}\right) \cap R\right)$. Moreover, one can take $R=K A^{*} K^{\prime}$ where $K$ and $K^{\prime}$ are proper regular languages defined on an alphabet $B$ disjoint from $A$.

Proof. Assume first that $\varepsilon \notin L$. Let $G=(N, A, P, S)$ be a type 0 grammar generating $L$, where we can assume that the productions have no terminal letters on the right hand side, i.e., $P \subseteq N^{+} \times(N \cup A)^{+}$. Let $\bar{A}$ be a copy of $A$ that is disjoint from the other alphabets. Also, let $V=N \cup \bar{A}$ and $R=K A^{*} K^{\prime}$ with

$$
K=S_{0} \triangleright\left(V^{*} P V^{*} \triangleright\right)^{*} \text { and } K^{\prime}=F \#^{*},
$$

where $S_{0}, \triangleright, F$ and \# are new symbols.

Let us define the morphisms $h_{1}$ and $h_{2}$ by

\begin{tabular}{c|c|c|c|c|c|c|c|c} 
& $S_{0}$ & $\triangleright$ & $p=(u, v)$ & $X \in N$ & $\bar{a} \in \bar{A}$ & $a \in A$ & $F$ & $\#$ \\
\hline$h_{1}$ & $S_{0} \triangleright S$ & $\triangleright$ & $v$ & $X$ & $a$ & $\#$ & $\#$ & $\#$ \\
$h_{2}$ & $S_{0}$ & $\triangleright$ & $u$ & $X$ & $a$ & $a$ & $\triangleright$ & $\# \#$
\end{tabular}

Let us take $u \in \pi_{A}\left(E\left(h_{1}, h_{2}\right) \cap R\right)$. Then there exists a word $z \in$ $E\left(h_{1}, h_{2}\right) \cap R$ such that $h_{1}(z)=h_{2}(z)$, and $u=\pi_{A}(z)$. Here

$$
z=S_{0} \triangleright z_{1} \triangleright \ldots \triangleright z_{n} F \#^{i}
$$


where $z_{1}, \ldots, z_{n-1} \in V^{*} P V^{*}, u=z_{n}$, and $i \geq 0$. Hence, for $1 \leq j \leq n-1$,

$$
\begin{aligned}
h_{2}\left(z_{j}\right) & \Longrightarrow{ }_{G} h_{1}\left(z_{j}\right), \\
h_{1}\left(S_{0} \triangleright z_{1} \triangleright \ldots \triangleright z_{j}\right) & =h_{2}\left(S_{0} \triangleright z_{1} \triangleright \ldots \triangleright z_{j+1}\right) .
\end{aligned}
$$

Therefore, $h_{2}\left(z_{1}\right)=S$ and $h_{1}\left(z_{j}\right)=h_{2}\left(z_{j+1}\right)$ for $1 \leq j \leq n-1$. So we obtain that

$$
\begin{gathered}
S=h_{2}\left(z_{1}\right) \Longrightarrow_{G} h_{1}\left(z_{1}\right)=h_{2}\left(z_{2}\right) \Longrightarrow_{G} \ldots \Longrightarrow_{G} h_{1}\left(z_{n-2}\right)= \\
=h_{2}\left(z_{n-1}\right) \Longrightarrow_{G} h_{1}\left(z_{n-1}\right)=h_{2}\left(z_{n}\right)=u
\end{gathered}
$$

and therefore $u \in L$.

Conversely, if $u \in L$, then we have a derivation

$$
S=w_{1} \Longrightarrow_{G} w_{2} \Longrightarrow_{G} \ldots \Longrightarrow_{G} w_{n}=u
$$

according to the grammar $G$. For each $1 \leq j \leq n-1$, one can find $z_{j} \in$ $V^{*} P V^{*}$ such that $h_{1}\left(z_{j}\right)=w_{j+1}$ and $h_{2}\left(z_{j}\right)=w_{j}$. Set then

$$
z=S_{0} \triangleright z_{1} \triangleright \ldots \triangleright z_{n-1} \triangleright u F \#^{i+1},
$$

where $i$ is the length of $u$. Then $z \in R$ and one can easily check that $h_{1}(z)=h_{2}(z)$. Hence, $u=\pi_{A}(z) \in \pi_{A}\left(E\left(h_{1}, h_{2}\right) \cap R\right)$.

Finally, if $\varepsilon \in L$, set $D=A \cup\{d\}$, where $d$ is a new symbol. Then

$$
L d=\pi_{D}\left(E\left(h_{1}, h_{2}\right) \cap K D^{*} K^{\prime}\right)=\pi_{D}\left(E\left(h_{1}, h_{2}\right) \cap K A^{*} d K^{\prime}\right)
$$

and hence $L=\pi_{A}\left(E\left(h_{1}, h_{2}\right) \cap K A^{*} d K^{\prime}\right)$. This completes the proof of the lemma.

Note that the form of the regular language $R$ and the fact that the two morphisms are nonerasing are crucial for the proofs of the following lemmata. The proof of the following lemma uses the methods from [9].

Lemma 11. Let $A$ and $B$ be two disjoint alphabets and $h_{1}, h_{2}:(A \cup B)^{*} \rightarrow$ $C^{*}$ be two nonerasing morphisms. If $K$ and $K^{\prime}$ are two regular languages included in $B^{+}$, then $\pi_{A}\left(E\left(h_{1}, h_{2}\right) \cap K A^{*} K^{\prime}\right)=\pi_{A}\left(E_{G}\left(\#, g_{1}, g_{2}\right)\right)$ for some nonerasing morphisms $g_{1}$ and $g_{2}$.

Proof. Let us take two nondeterministic finite automata $M=\left(Q, B, \Delta, q_{0}, F\right)$, $M^{\prime}=\left(Q^{\prime}, B, \Delta^{\prime}, q_{0}^{\prime}, F^{\prime}\right)$ such that $L(M)=K$ and $L\left(M^{\prime}\right)=K^{\prime}$. The transitions are triples of the form $(q, b, p)$, that is, $\Delta \subseteq Q \times B \times Q$ and $\Delta^{\prime} \subseteq Q^{\prime} \times B \times Q^{\prime}$. Clearly, one can assume that $Q=\left\{q_{0}, \ldots, q_{n}\right\}$ and $Q^{\prime}=\left\{q_{0}^{\prime}, \ldots, q_{n}^{\prime}\right\}$ with $Q \cap Q^{\prime}=\emptyset$, and that the automata have unique final states $F=\left\{q_{n}\right\}$ and $F^{\prime}=\left\{q_{n}^{\prime}\right\}$. Also, we can assume that there are no transitions $\left(q, b, q_{0}\right)$ and $\left(q^{\prime}, b, q_{0}^{\prime}\right)$ that enter the initial states $q_{0}$ and $q_{0}^{\prime}$, and 
that there are no transitions $\left(q_{f}, b, q\right)$ and $\left(q_{f}^{\prime}, b, q^{\prime}\right)$ leaving from the final states $q_{f}$ and $q_{f}^{\prime}$.

First, we define three morphisms $\Theta, \ell$ and $r$ as follows.

Let $\theta:\left(A \cup \Delta \cup \Delta^{\prime}\right)^{*} \rightarrow(A \cup B)^{*}$ be the morphism defined by

$$
\theta(a)=a \text { for } a \in A \text {, and } \theta((p, b, q))=b \text { for }(p, b, q) \in \Delta \cup \Delta^{\prime} .
$$

Also, let $\ell=\ell_{d^{2 n}}$ and $r=r_{d^{2 n}}$, that is, $\ell, r: C^{*} \rightarrow(C \cup\{d\})^{*}$, where $d$ is a new symbol, and

$$
\ell(c)=d^{2 n} c, \text { and } r(c)=c d^{2 n} \text { for } c \in C .
$$

The morphism $g_{2}:\left(A \cup \Delta \cup \Delta^{\prime}\right)^{*} \rightarrow(C \cup\{\#, d\})^{*}$ becomes defined by

$$
g_{2}=r h_{2} \theta \text {. }
$$

It is immediate that $g_{2}(z) \in\left(C d^{2 n}\right)^{*}$ for all $z \in\left(A \cup \Delta \cup \Delta^{\prime}\right)^{*}$. The shift letter \# does not belong to any image of $g_{2}$. The notation $w d^{-m}$ means $w\left(d^{m}\right)^{-1}$, that is, $d^{m}$ is removed as a suffix of the word $w$, and similarly $d^{-m} w$ is an abbreviation for $\left(d^{m}\right)^{-1} w$. The morphism $g_{1}:\left(A \cup \Delta \cup \Delta^{\prime}\right)^{*} \rightarrow(C \cup\{\#, d\})^{*}$ is defined by

$$
\begin{aligned}
& g_{1}\left(\left(q_{0}, b, q_{j}\right)\right)=\# r h_{1}(b) d^{-2 j}, \\
& g_{1}\left(\left(q_{i}, b, q_{j}\right)\right)=d^{2 i} r h_{1}(b) d^{-2 j} \text { for } i \neq 0, \\
& g_{1}(a)=\ell h_{1}(a) \text { for } a \in A, \\
& g_{1}\left(\left(q_{0}^{\prime}, b, q_{n}^{\prime}\right)\right)=\ell h_{1}(b) d^{2 n}, \\
& g_{1}\left(\left(q_{0}^{\prime}, b, q_{j}^{\prime}\right)\right)=\ell h_{1}(b) d^{2 j+1} \text { for } j \neq n, \\
& g_{1}\left(\left(q_{i}^{\prime}, b, q_{n}^{\prime}\right)\right)=d^{-(2 i+1)} \ell h_{1}(b) d^{2 n} \text { for } i \neq 0, \\
& g_{1}\left(\left(q_{i}^{\prime}, b, q_{j}^{\prime}\right)\right)=d^{-(2 i+1)} \ell h_{1}(b) d^{2 j+1} \text { for } i \neq 0 \text { and } j \neq n .
\end{aligned}
$$

The morphism $g_{1}$ decodes the behaviour of the combined automata that accepts the language $K A^{*} K^{\prime}$ in the sense that $g_{1}(z) \in \#\left(C d^{2 n}\right)^{*}$ if and only if $z=u v u^{\prime}$ for some words $u \in \Delta^{*}, v \in A^{*}$, and $u^{\prime} \in \Delta^{* *}$ such that $\theta(u) \in K$ and $\theta\left(u^{\prime}\right) \in K^{\prime}$. Therefore, we have

$$
g_{1}(z) \in \#\left(C d^{2 n}\right)^{*} \Longleftrightarrow \theta(z) \in K A^{*} K^{\prime} .
$$

Finally, let $\pi=\pi_{C}$ be the projection $\pi:(C \cup\{\#, d\})^{*} \rightarrow C^{*}$ that deletes the letters $d$ and \#. Then we have

$$
\pi g_{1}=h_{1} \theta \text { and } \pi g_{2}=h_{2} \theta .
$$

Let $v$ be a word in $\pi_{A}\left(E_{G}\left(\#, g_{1}, g_{2}\right)\right)$ and let $z$ be such that $v=\pi_{A}(z)$ and $g_{1}(z)=\# g_{2}(z)$. Since $g_{2}(z) \in\left(C d^{2 n}\right)^{*}$, also $g_{1}(z) \in\left(C d^{2 n}\right)^{*}$, and it follows by (4.1) that $\theta(z) \in K A^{*} K^{\prime}$. Consequently, by (4.2), we have

$$
h_{1} \theta(z)=\pi g_{1}(z)=\pi\left(\# g_{2}(z)\right)=\pi g_{2}(z)=h_{2} \theta(z) .
$$


Hence, $\theta(z) \in E\left(h_{1}, h_{2}\right) \cap K A^{*} K^{\prime}$ and also $v=\pi_{A} \theta(z) \in \pi_{A}\left(E\left(h_{1}, h_{2}\right) \cap\right.$ $\left.K A^{*} K^{\prime}\right)$ as required.

Conversely, let $v \in \pi_{A}\left(E\left(h_{1}, h_{2}\right) \cap K A^{*} K^{\prime}\right)$, say $v=\pi_{A}\left(k v k^{\prime}\right)$ with $k \in K, k^{\prime} \in K^{\prime}$ and $h_{1}\left(k v k^{\prime}\right)=h_{2}\left(k v k^{\prime}\right)$. Then there exists a word $z=u v u^{\prime}$ with $u \in \Delta^{+}, u^{\prime} \in \Delta^{\prime+}, \theta(u)=k, \theta\left(u^{\prime}\right)=k^{\prime}, \theta(z)=k v k^{\prime}$ and $g_{1}(z)=$ $\# r h_{1} \theta(z)=\# r h_{2} \theta(z)=\# g_{2}(z)$. Therefore, $v=\pi_{A}(z) \in \pi_{A}\left(E_{G}\left(\#, g_{1}, g_{2}\right)\right)$, which completes the proof.

From the two above lemmata, we obtain immediately the following result.

Theorem 12. Every recursively enumerable language $L \subseteq A^{*}$ is a projection of a shifted equality set, that is, $L=\pi_{A}\left(E_{G}\left(a, g_{1}, g_{2}\right)\right)$ for a letter a and some nonerasing morphisms $g_{1}$ and $g_{2}$.

We remark that from this result it is very easy to find again Theorem 9 . Indeed, if $L=\pi_{A}\left(E_{G}\left(a, g_{1}, g_{2}\right)\right)$ for some morphisms $g_{1}$ and $g_{2}$ defined on an alphabet $X$, one gets $L=\pi_{A}\left(E\left(h_{1}, h_{2}\right) \cap d X^{*}\right)$ where $d$ is a new letter, $h_{1}(d)=d, h_{2}(d)=d a$ and $h_{i}(x)=g_{i}(x)$ for $x \in X$. Note also that the regular language $d X^{*}$ is quite simple!

Two morphisms, $g_{1}, g_{2}: A^{*} \rightarrow B^{*}$ are said to be prefix-incomparable, if for each letter $a \in A, g_{1}(a)$ is not a prefix of $g_{2}(a)$ and $g_{2}(a)$ is not a prefix of $g_{1}(a)$.

Lemma 13. Let $L=E_{G}\left(\#, h_{1}, h_{2}\right)$ where $h_{1}$ and $h_{2}$ are nonerasing morphisms defined on the alphabet $A$. Then $L=\pi_{A}\left(E_{G}\left(\#, g_{1}, g_{2}\right)\right)$ for some prefix-incomparable nonerasing morphisms $g_{1}$ and $g_{2}$.

Proof. Let $h_{1}, h_{2}: A^{*} \rightarrow X^{*}$, and let $c$ and $d$ be new letters. Set $B=A \cup\{d\}$ and $Y=X \cup\{c, d\}$. Recall that $\ell_{c}, r_{c}: X^{*} \rightarrow Y^{*}$ are defied by $\ell_{c}(x)=c x$ and $r_{c}(x)=x c$. Let $g_{1}: B^{*} \rightarrow Y^{*}$ and $g_{2}: B^{*} \rightarrow Y^{*}$ be defined by

$$
\begin{aligned}
& g_{1}(d)=d \text { and } g_{1}(a)=r_{c} h_{1}(a) \text { for } a \in A \\
& g_{2}(d)=c d \text { and } g_{2}(a)=\ell_{c} h_{2}(a) \text { for } a \in A
\end{aligned}
$$

Clearly, $g_{1}(b)$ and $g_{2}(b)$ are prefix-incomparable morphisms. We have, for each $u \in A^{*}$, that

$$
\# g_{2}(u d)=\# g_{2}(u) c d=\# \ell h_{2}(u) c d=r\left(\# h_{2}(u)\right) d .
$$

Now, if $u \in L$, that is, $h_{1}(u)=\# h_{2}(u)$, then it follows from (4.3) that $\# g_{2}(u d)=r\left(\# h_{2}(u)\right) d=r h_{1}(u)=g_{1}(u d)$. Hence $u=\pi_{A}(u d) \in \pi_{A}\left(E_{G}\left(\#, g_{1}, g_{2}\right)\right)$.

Conversely, assume that $u \in \pi_{A}\left(E_{G}\left(\#, g_{1}, g_{2}\right)\right)$. Then there exists a word $v$ such that $u=\pi_{A}(v)$ and $g_{1}(v)=\# g_{2}(v)$. By the definitions of the morphisms $g_{1}$ and $g_{2}$, we must have $v=u d$. From (4.3), we obtain $\# g_{2}(v)=$ $r\left(\# h_{2}(u)\right) d=g_{1}(v)=g_{1}(u d)=r h_{1}(u) d$. Thus $r\left(\# h_{2}(u)\right) d=r\left(h_{1}(u)\right)$, which implies $\# h_{2}(u)=h_{1}(u)$ and $u \in E_{G}\left(\#, h_{1}, h_{2}\right)$ as required. 
A language $L \subseteq A^{*}$ is a star language, if $L=L^{*}$, that is, if it is closed under concatenation.

As seen in the preliminaries, equality sets are star languages. So it is clear that projections of equality sets are recursively enumerable star languages. As a matter of fact, the following result shows that these two families coincide.

Theorem 14. Every recursively enumerable star language is a projection of an equality set, that is, for every recursively enumerable $L \subseteq A^{*}$, there are nonerasing morphisms $g_{1}$ and $g_{2}$ such that $L^{*}=\pi_{A}\left(E\left(g_{1}, g_{2}\right)\right)$.

Proof. From Theorem 12, we have that $L^{*}=\pi_{A}\left(E_{G}\left(\#, h_{1}, h_{2}\right)\right)$ for some nonerasing morphisms $h_{1}$ and $h_{2}$ defined on an alphabet $X$. When we apply Lemma 13 to the shifted equality set $E_{G}\left(\#, h_{1}, h_{2}\right)$, we can, without loss of generality, assume that the morphisms $h_{1}$ and $h_{2}$ prefix-incomparable. Let $d$ be a new letter and set $Y=X \cup\{d\}$. Let us define the morphisms $g_{1}$ and $g_{2}$ by:

$$
\begin{aligned}
& g_{1}(d)=d \quad \text { and } \quad g_{1}(x)=h_{1}(x) \text { for } x \in X \\
& g_{2}(d)=d \# \text { and } g_{2}(x)=h_{2}(x) \text { for } x \in X
\end{aligned}
$$

Now, if $u \in L^{*}$, we have $g_{1}(d u)=d h_{1}(u)=d \# h_{2}(u)=g_{2}(d u)$ and from (4.3) $\# g_{2}(u d)=r\left(\# h_{2}(u)\right) d=r h_{1}(u)=g_{1}(u d)$. Hence $u \in \pi_{A}\left(E\left(g_{1}, g_{2}\right)\right)$.

Conversely, let $u \in \pi_{A}\left(E\left(g_{1}, g_{2}\right)\right)$. Then $u=\pi_{A}(v)$ with $g_{1}(v)=g_{2}(v)$. Since for each $x \in X, h_{1}(x)$ and $h_{2}(x)$ are prefix-incomparable, we have $v=d v_{1} \ldots d v_{n}$ where each $v_{i}$ is in $X^{*}$. Now,

$$
g_{1}(v)=d h_{1}\left(v_{1}\right) \ldots d h_{1}\left(v_{n}\right)=g_{2}(v)=d \# h_{2}\left(v_{1}\right) \ldots d \# h_{2}\left(v_{n}\right) .
$$

Therefore $h_{1}\left(v_{i}\right)=\# h_{2}\left(v_{i}\right)$ for each $i$, and $\pi_{A}\left(v_{i}\right) \in L^{*}$. From these we obtain $u=\pi_{A}(v)=\pi_{A}\left(d v_{1} \ldots d v_{n}\right)=\pi_{A}\left(v_{1} \ldots v_{n}\right) \in L^{*}$, which proves the claim.

We conclude with a remark that Theorem 9 is a direct consequence of this result. Indeed, let $L \subseteq A^{*}$ be a recursively enumerable language, $d$ a new letter and set $D=A \cup\{d\}$. From Theorem 14, we obtain $(L d)^{*}=$ $\pi_{D}\left(E\left(g_{1}, g_{2}\right)\right)$, and hence

$$
L d=(L d)^{*} \cap A^{*} d=\pi_{D}\left(E\left(g_{1}, g_{2}\right) \cap \pi_{D}^{-1}\left(A^{*} d\right)\right)
$$

and so $L=\pi_{A}\left(E\left(g_{1}, g_{2}\right) \cap \pi_{D}^{-1}\left(A^{*} d\right)\right)$ as required.

\section{References}

[1] K. Culik II, A purely homomorphic characterization of recursively enumerable sets, J. Assoc. Comput. Mach. 26 (1979), 345-350. 
[2] J. Engelfriet and G. Rozenberg, Equality languages and fixed point languages, Inform. Control 43 (1979), 20-49.

[3] J. Engelfriet and G. Rozenberg, Fixed point languages, equality languages, and representation of recursively enumerable languages, J. Assoc. of Comput. Mach. 27 (1980), 499-518.

[4] V. Geffert, A representation of recursively enumerable languages by two homomorphisms and a quotient, Theoret. Comput. Sci. 62 (1988), 235249 .

[5] S. Ginsburg, Algebraic and Automata-theoretic Properties of Formal Languages, North-Holland, 1975.

[6] V. Halava, T. Harju, H. J. Hoogeboom, and M. Latteux, Valence Languages Generated by Generalized Equality Sets, J. Autom. Lang. Comb., to appear.

[7] V. Halava, T. Harju, H. J. Hoogeboom, and M. Latteux, Languages defined by generalized equality sets, 14th Internat. Symp. on Fundamentals of Computation Theory (FCT'03, Malmö, Sweden, 2003; A. Lingas, B.J. Nilsson, eds.), Lecture Notes in Comput. Sci. 2751 (2003), 355-363.

[8] T. Harju and J. Karhumäki, Morphisms, Handbook of Formal Languages (G. Rozenberg and A. Salomaa, eds.), vol. 1, Springer-Verlag, 1997.

[9] M. Latteux and J. Leguy, On the composition of morphisms and inverse morphisms, Lecture Notes in Comput. Sci. 154 (1983), 420-432.

[10] M. Latteux and J. Leguy, On usefulness of bifaithful rational cones, Math. Systems Theory 18 (1985), 19-32.

[11] M. Latteux and P. Turakainen, On characterization of recursively enumerable languages, Acta Informatica 28 (1990), 179-186.

[12] Gh. Păun, A new generative device: valence grammars, Revue Roumaine de Math. Pures et Appliquées 6 (1980), 911-924.

[13] A. Salomaa, Formal Languages, Academic Press, New York, 1973.

[14] A. Salomaa, Equality sets for homomorphisms of free monoids, Acta Cybernetica 4 (1978), 127-139.

[15] A. Salomaa, Jewels of Formal Language Theory, Computer Science Press, 1981.

[16] P. Turakainen, A unified approach to characterizations of recursively enumerable languages, Bulletin of the EATCS 45 (1991), 223-228. 

Turku Centre for Computer Science

Lemminkäisenkatu 14

FIN-20520 Turku

Finland

http://www.tucs.fi

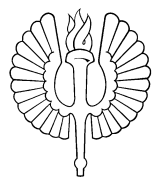

\author{
University of Turku \\ - Department of Information Technology \\ - Department of Mathematics
}

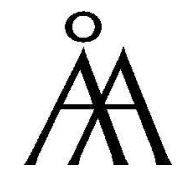

Åbo Akademi University

- Department of Computer Science

- Institute for Advanced Management Systems Research

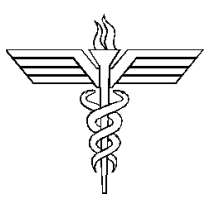

Turku School of Economics and Business Administration

- Institute of Information Systems Science 